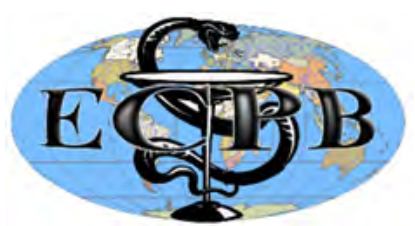

"ЕКСПЕРИМЕНТАЛЬНА ТА КЛІНІЧНА ФІЗІОЛОГІЯ І БІОХІМІЯ" "EXPERIMENTAL AND CLINICAL PHYSIOLOGY AND BIOCHEMISTRY" Науково-практичний журнал/Scientific-practical journal

Наукові статті / Research article ECPB 2018, 2(82): 21-30. https://doi.org/

\title{
Features of Metabolic Reactions to Various Water-Salt Loads in Female Rats
}

\author{
Y.V. ZAVIDNYUK \\ IY Horbachevs'kyi State Medical University, Ternopil', Ukraine, \\ Ukrainian Scientific Research Institute of Transport Medicine, Odesa, Ukraine \\ E-mail: zavidnyukyv@tdmu.edu.ua
}

Introduction. In the previous article [14], it has been reported that registered parameters of water-salt, nitrous and lipid metabolism as well as the neuroendocrine-immune complex of 42 rats subjected to various water-salt loads, significantly differ from those of the intact rats, these groups of animals have received liquids with different mineralization and chemical composition. The most heavily grown is glomerular filtration and mineralocorticoid activity, which is evaluated by the exchange of sodium and potassium, the activity of catalase plasma and urine, as well as the plasma testosterone, urea and malonic dialdehyde levels. Further, the following processes occur: urine excretion of calcium and associated with it and calciumemia calcitonin activity, as well as excretion of creatinine, magnesium and urea, concentration of creatinine in urine and plasma, urea concentration in urine and plasma glucose. The amount of thymocytogram of endothelial cytes and the Hassall's body have increased but in the splenocytogram reticulocytes index of killing by neutrophils Staph. aureus. In addition, diuresis, adrenals mass and triiodothyronine levels have increased. Instead, the mass of the spleen, the relative amount of the epitheliocytes, lymphoblasts and lymphocytes in the thymocytogram, eosinophils and stub neutrophils in the blood and splenocytogram plasmocytes have decreased as well as microbial number of neutrophils. The concentration of medium mass molecules and potassium in urine, have decreased. The maximum level of potassium and calcium in plasma has been reduced. Thus, a nonspecific (general) reaction of neuroendocrine-immune complex and metabolism in water-salt load has taken place despite of that fact that the specific chemical composition of fluids have been applied.

After these parameters have been revealed the changes which are common to the water-salt loads of different chemical composition, the specific manifestations of balneo reaction have been considered. The purpose of this article is to find out the features of the parameters the reactions of metabolism.

Material and Methods. Experiment has been performed at 58 healthy female Wistar rats 240-290g divided into 6 groups. Animals of the first group have remained intact because of using tap water from drinking ad libitum. Rats of the second (control) group have been administered a single tap water through the probe at a dose of $1,5 \mathrm{~mL} / 100 \mathrm{~g}$ of body mass during 6 days. The third group (reference for the organic component) has been given daily drinking water 'Naftussia' from the Truskavets' layer, the rats of the fourth group (reference to the salt component) have been given water Sophiya of the Truskavets' field. The rats of the main group have received 'Gertsa' water (Bukovyna) field, and the second control group - its artificial salt analogue. The

(C) Zavidnyk Y.V., 2018 
chemical composition of the applied samples of water (according to Truskavets Hydrological Regime-operational Station data) is depicted in Table 1.

The chemical composition of the applied mineral waters

\begin{tabular}{|l|c|c|c|c|c|}
\hline & Daily Water & Sofiya & Gertsa & Salt analog & Naftussya \\
\hline \multicolumn{7}{|c|}{ Electrolytes, mM/L } \\
\hline $\mathrm{Na}^{+}$ & 0,5 & 156 & 196,7 & 196,7 & 0,6 \\
\hline $\mathrm{Cl}$ & 3,4 & 142 & 205 & 205 & 1,0 \\
\hline $\mathrm{HCO}_{3}{ }^{-}$ & 2,9 & 7,5 & 5,6 & 5,6 & 8,2 \\
\hline $\mathrm{Ca}^{2+}$ & 3,4 & 5,3 & 3,40 & 3,40 & 2,9 \\
\hline $\mathrm{Mg}^{2+}$ & 0,5 & 4,3 & 3,44 & 3,44 & 2,3 \\
\hline $\mathrm{K}^{+}$ & 0,4 & 0,3 & 0,4 & 0,4 & 0,3 \\
\hline $\mathrm{SO}_{4}{ }^{2-}$ & 1,2 & 13,1 & 0,1 & 0,1 & 1,0 \\
\hline \multicolumn{7}{|c|}{ Trace elementes, mg/L } \\
\hline $\mathrm{H}_{2} \mathrm{SiO}_{3}$ & 5 & 4,43 & 9,88 & 0 & 9,5 \\
\hline $\mathrm{H}_{3} \mathrm{BO}_{3}$ & 0,25 & 8,39 & 42,76 & 0 & 0,200 \\
\hline $\mathrm{Br}$ & 8,3 & 6,7 & 21,17 & 0 & 0,034 \\
\hline $\mathrm{J}$ & 0,025 & 1,29 & 6,62 & 0 & 0,004 \\
\hline $\mathrm{F}$ & 0,95 & 0,52 & 0,57 & 0 & 0,160 \\
\hline \multicolumn{7}{|c|}{ Organic substances, mg/L } \\
\hline $\mathrm{C}$ org & 5,0 & 5,5 & 34 & 0 & 12,8 \\
\hline $\mathrm{N}$ org & 0,02 & 0,8 & 0,14 & 0 & 0,33 \\
\hline
\end{tabular}

The day after the completion of the drinking course animals have been placed into individual chambers with perforated bottom for collecting daily urine. The experiment has been completed by decapitation of rats in order to collect as much blood as possible.

Plasma levels of the electrolytes have been determined: calcium (by reaction with arsenic III), magnesium (by reaction with colgamite), phosphates (phosphate-molybdate method), chloride (mercury-rhodanine method), sodium and potassium (both in plasma and in erythrocytes) by flamming photometry; nitric metabolites: creatinine (by Jaffe's color reaction by Popper's method), urea (urease method by reaction with phenol hypochlorite), uric acid (uricase method), medium molecular polypeptides (by spectrophotometric method), bilirubin (by diazo reaction using the Jedrasik-Cleghorne-Grof method); lipid peroxidation products: diene conjugates (spectrophotometry of the heptane phase of the lipids extract) and malonic dialdehyde (in the test with thiobarbituric acid), antioxidant enzymes: superoxide dismutase erythrocytes (according to the degree of inhibition of reduction of nitroblue tetrazolium in the presence of $\mathrm{N}$-methylphenazinium methosulfate and NADH) and catalase plasma (at the rate of decomposition of hydrogen peroxide), as well as amylase (Karavay's amyloclastic method with starch substrate) and glucose (glucose-oxidase method).

Most of the listed parameters of metabolism have been also detected in the daily urine. Glomerular filtration and tubular reabsorption have been calculated by the size of the diuresis and the level of creatinine in plasma and urine. In addition, the osmolarity of the urine has been measured by the cryostatic method.

The analyzes have been carried out according to the instructions described in the manual [4]. The analyzers "Pointe-180" ("Scientific", USA) and "Reflotron" (Boehringer Mannheim, BRD)with the appropriate sets and a flaming spectrophotometer "C $\Phi-47$ " have been used.

Digital material is statistically processed on a computer using the software package "Statistica 5.5". 
Results and Discusion. In the first stage of the analysis, all registered parameters have been divided into seven patterns. The pattern is a characteristic sequence of localization of rats in a plane whose $\mathrm{Y}$ axis represents the mean of the Z-scores. At the second stage of the analysis, the quasi-mirror patterns have been paired.

The first pattern (Table 2 and Fig. 1) combines nine parameters, the average Z-scores which is maximum for the rats that have received artificial saline analogue of mineral water "Gertsa". Next, the therapeutic water "Sofiya" and "Naftussia", as well as the daily water effects of which on these parameters are approximately equally moderate.

Significantly, glomerular filtration and excretion of creatinine and urea, as well as diuresis are the most likely to increase. Back in 2004, A.I. Gozhenko et al. [5] showed that one-time loading with $0,5 \%$ solution $\mathrm{NaCl}(5 \mathrm{~mL} / \mathrm{kg}$ of body mass) as compared with daily water load is immediately followed by reliably increased excretion of creatinine and nitrous metabolites at healthy volunteers. A simple recount shows that in the water "Gertsa" the concentration of $\mathrm{NaCl}$ is $1,28 \%$ and in the water "Sofiya" is $0,86 \%$. Nevertheless, their influence on these parameters of the kidneys is much weaker, due, apparently, to the presence of sulphate and/or of trace elements and organic substances in their composition. Despite the expectations and extensive literature [1-3, 6-13], the influence of 'Naftussia' water on diuresis and urine excretion of metabolites has been weaker.

The second pattern combines seven parameters, the mean Z-score of which is minimal in similar conditions, while under the influence of other loads, they decrease to a lesser extent. First of all, it is the concentration of potassium, phosphates and medium molecules in the urine.

Configurations of patterns are almost specular, that has served as the basis for their visualization on a common plane.

Table 2

The first pair of patterns of reactions of metabolic parameters to water-salt loadings

\begin{tabular}{|l|c|c|c|c|c|c|}
\hline \multicolumn{1}{|c|}{ Variables } & $\begin{array}{c}\text { Salt } \\
\text { Anal } \\
\text { G (8) }\end{array}$ & $\begin{array}{c}\text { MW } \\
\text { Gertsa } \\
\mathbf{( 1 1 )}\end{array}$ & $\begin{array}{c}\text { MW } \\
\text { Sofiya } \\
\mathbf{( 1 0 )}\end{array}$ & $\begin{array}{c}\text { Naftu } \\
\text { ssya } \\
\mathbf{( 9 )}\end{array}$ & $\begin{array}{c}\text { Daily } \\
\text { Water } \\
\mathbf{( 1 0 )}\end{array}$ & $\begin{array}{c}\text { Intact } \\
\text { rats } \\
\mathbf{( 1 0 )}\end{array}$ \\
\hline Glomerulary Filtration, & 194 & 142 & 112 & 109 & 86,5 & 85,9 \\
$\mu \mathrm{L} / \mathrm{min} \cdot 100$ g Body Mass & 2,26 & 1,65 & 1,30 & 1,27 & 1,01 & 1 \\
& $+1,75$ & $+0,91$ & $+0,41$ & $+0,38$ & $+0,01$ & 0 \\
\hline Creatinine Excretion, & 16,03 & 10,53 & 12,30 & 12,27 & 10,12 & 8,72 \\
$\mu \mathrm{M} / 24 \mathrm{~h} \cdot 100$ g Body Mass & 1,84 & 1,21 & 1,41 & 1,41 & 1,16 & 1 \\
& $+1,68$ & $+0,42$ & $+0,82$ & $+0,82$ & $+0,32$ & 0 \\
\hline Katalase & 163 & 141 & 136 & 145 & 151 & 123 \\
Urine, & 1,33 & 1,15 & 1,11 & 1,18 & 1,23 & 1 \\
$\mathrm{nM} / \mathrm{h} \cdot \mathrm{mL}$ & $+1,47$ & $+0,65$ & $+0,48$ & $+0,80$ & $+1,04$ & 0 \\
\hline $\mathrm{Urea}$ Excretion, & 315 & 283 & 192 & 201 & 164 & 169 \\
$\mathrm{\mu M} / 24 \mathrm{~h} \cdot 100$ g Body Mass & 1,86 & 1,68 & 1,14 & 1,19 & 0,97 & 1 \\
& $+1,08$ & $+0,85$ & $+0,17$ & $+0,24$ & $-0,04$ & 0 \\
\hline Diurese, & 2,37 & 1,66 & 1,53 & 1,65 & 1,44 & 1,44 \\
$\mathrm{~mL} / 24 h \cdot 100$ g Body Mass & 1,65 & 1,15 & 1,06 & 1,14 & 1,00 & 1 \\
& $+1,05$ & $+0,25$ & $+0,10$ & $+0,23$ & 0,00 & 0 \\
\hline Diene conjugates & 1,63 & 1,65 & 1,30 & 1,31 & 1,45 & 1,35 \\
Plasma, & 1,21 & 1,23 & 0,96 & 0,97 & 1,08 & 1 \\
E232/mL & $+0,72$ & $+0,76$ & $-0,13$ & $-0,10$ & $+0,25$ & 0 \\
\hline Glukose & 5,76 & 5,15 & 5,31 & 5,32 & 5,61 & 4,95 \\
Plasma, & 1,16 & 1,04 & 1,07 & 1,08 & 1,13 & 1 \\
mM/L & $+0,74$ & $+0,19$ & $+0,33$ & $+0,34$ & $+0,60$ & 0 \\
\hline
\end{tabular}



https://doi.org/

\begin{tabular}{|l|c|c|c|c|c|c|}
\hline Phosphates Excretion, & 137 & 108 & 92 & 104 & 93 & 94 \\
$\mu \mathrm{MM} / 24 \mathrm{~h} \cdot 100$ g Body Mass & 1,46 & 1,15 & 0,98 & 1,11 & 0,99 & 1 \\
& $+0,69$ & $+0,23$ & $-0,03$ & $+0,16$ & $-0,01$ & 0 \\
\hline Urea & 129 & 141 & 122 & 118 & 104 & 107 \\
Urine, & 1,20 & 1,32 & 1,14 & 1,10 & 0,97 & 1 \\
$\mathrm{mM} / \mathrm{L}$ & $+0,52$ & $+0,83$ & $+0,36$ & $+0,27$ & $-0,08$ & 0 \\
\hline Pattern I (9) & $+1,08$ & $+0,57$ & $+0,28$ & $+0,35$ & $+0,23$ & 0 \\
& $\pm 0,15$ & $\pm 0,10$ & $\pm 0,10$ & $\pm 0,10$ & $\pm 0,13$ & \\
\hline Potassium & 95 & 122 & 128 & 104 & 125 & 131 \\
Urine, & 0,73 & 0,93 & 0,98 & 0,79 & 0,96 & 1 \\
mM/L & $-0,91$ & $-0,23$ & $-0,08$ & $-0,70$ & $-0,14$ & 0 \\
\hline Middle Mass Molecules & 147 & 165 & 159 & 159 & 181 & 182 \\
Urine, & 0,80 & 0,91 & 0,87 & 0,87 & 0,99 & 1 \\
units & $-0,68$ & $-0,32$ & $-0,44$ & $-0,44$ & $-0,02$ & 0 \\
\hline Phosphates & 58,9 & 62,6 & 61,3 & 63,8 & 63,5 & 63,9 \\
Urine, & 0,92 & 0,98 & 0,96 & 1,00 & 0,99 & 1 \\
mM/L & $-0,64$ & $-0,16$ & $-0,33$ & $-0,01$ & $-0,05$ & 0 \\
\hline Potassium & 3,82 & 3,35 & 3,12 & 3,86 & 3,71 & 4,23 \\
Plasma, & 0,90 & 0,79 & 0,74 & 0,91 & 0,88 & 1 \\
mM/L & $-0,58$ & $-1,25$ & $-1,58$ & $-0,53$ & $-0,73$ & 0 \\
\hline Superoxide Dismutase & 53,3 & 51,4 & 57,8 & 56,8 & 56,3 & 58,0 \\
Plasma, & 0,92 & 0,89 & 1,00 & 0,98 & 0,97 & 1 \\
units/mL & $-0,44$ & $-0,62$ & $-0,02$ & $-0,12$ & $-0,16$ & 0 \\
\hline Superoxide Dismutase & 59,9 & 59,8 & 62,6 & 59,6 & 62,7 & 61,3 \\
Urine, & 0,98 & 0,97 & 1,02 & 0,97 & 1,02 & 1 \\
units/mL & $-0,27$ & $-0,29$ & $+0,24$ & $-0,33$ & $+0,26$ & 0 \\
\hline Bilirubine & 3,94 & 4,77 & 4,20 & 4,70 & 5,04 & 4,63 \\
Plasma, & 0,85 & 1,03 & 0,91 & 1,02 & 1,09 & 1 \\
pM/L & $-0,27$ & $+0,06$ & $-0,17$ & $+0,03$ & $+0,16$ & 0 \\
\hline Pattern II (7) & $-0,54$ & $-0,40$ & $-0,34$ & $-0,30$ & $-0,10$ & 0 \\
& $\pm 0,09$ & $\pm 0,16$ & $\pm 0,22$ & $\pm 0,10$ & $\pm 0,12$ & \\
\hline
\end{tabular}

Notes.1. In each graph, the actual mean values are in the first line, the average values of the intact group (L/I) are in the second line, $\mathrm{Z}=(\mathrm{L} / \mathrm{I}-1) / \mathrm{Cv}$ is in the third row.

2. Patterns display average values of $\mathrm{Z}$ and their standard errors.

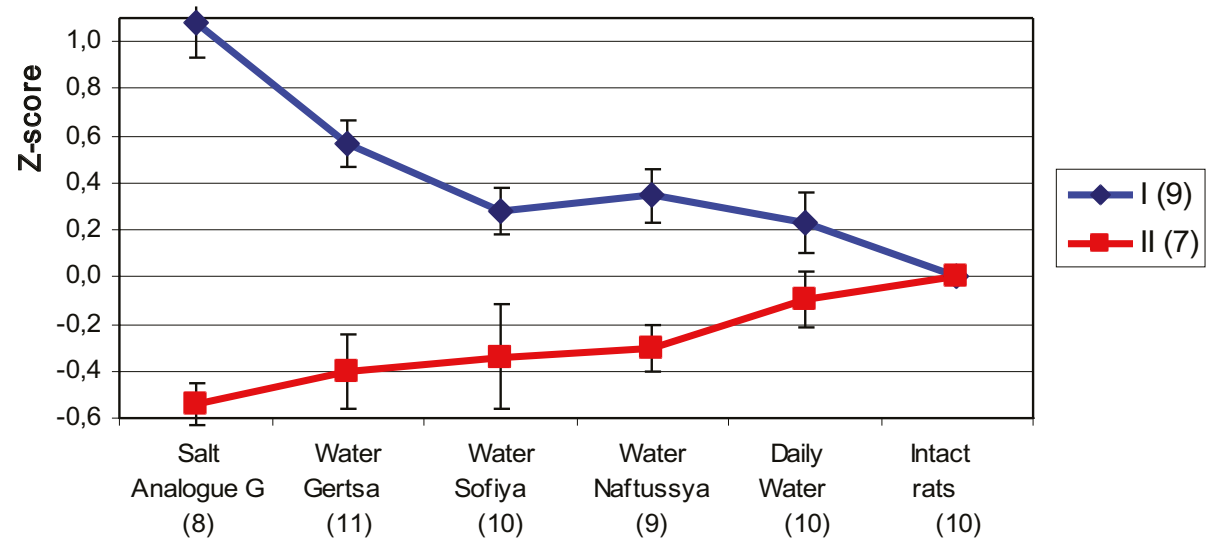

Fig. 1. The first pair of patterns of reactions of metabolic parameters to water-salt loads.

The second pattern (Table 3 and Fig. 2) combines 12 parameters, the mean of Z-score which is reduced to a greater extent under the influence of bioactive water 'Naftussia' and less noticeable in the rats receiving daily water from the 
tap, while the tendency towards increasing is detected in the animals that have received the mineral water "Sofiya" and "Gertsa" and have significantly increased under the influence of the salt analogue of the latter.

Mostly, the excretion and concentration of sodium and chloride in urine decrease, this leads to the decrease of the osmolarity of the urine. In addition, diene conjugates in the urine, uric acid and calcium in the plasma, sodium and potassium in erythrocytes are reduced, as well as tubular reabsorption of water.

On the contrary, the opposite changes in these parameters are reflected with the exception of a significant increase of chloride and potassium in urinary concentration, the urinary osmolality caused by these ions and the concentration in calcium plasma.

Table 3

The second pair of patterns of reactions of metabolic parameters to water-salt loadings

\begin{tabular}{|c|c|c|c|c|c|c|}
\hline Variables & $\begin{array}{l}\text { Salt } \\
\text { Anal } \\
\text { G (8) }\end{array}$ & $\begin{array}{l}\text { MW } \\
\text { Gertsa } \\
\text { (11) }\end{array}$ & $\begin{array}{l}\text { MW } \\
\text { Sofiya } \\
(10)\end{array}$ & $\begin{array}{l}\text { Intact } \\
\text { rats } \\
(10)\end{array}$ & $\begin{array}{l}\text { Daily } \\
\text { Water } \\
(10)\end{array}$ & $\begin{array}{l}\text { Naftu } \\
\text { ssya } \\
\text { (9) }\end{array}$ \\
\hline $\begin{array}{l}\text { Osmolarity } \\
\text { Urine, } \\
\text { mOsm/L }\end{array}$ & $\begin{array}{c}581 \\
1,04 \\
+0,16 \\
\end{array}$ & $\begin{array}{c}623 \\
1,11 \\
+0,46 \\
\end{array}$ & $\begin{array}{c}598 \\
1,07 \\
+0,28\end{array}$ & $\begin{array}{c}559 \\
1 \\
0\end{array}$ & $\begin{array}{c}464 \\
0,83 \\
-0,69\end{array}$ & $\begin{array}{c}424 \\
0,76 \\
-0,98\end{array}$ \\
\hline $\begin{array}{l}\text { Sodium Excretion, } \\
\mu \mathrm{M} / 24 \mathrm{~h} \cdot 100 \mathrm{~g} \text { Body Mass }\end{array}$ & $\begin{array}{c}282 \\
2,09 \\
+1,75 \\
\end{array}$ & $\begin{array}{r}225 \\
1,67 \\
+1,08 \\
\end{array}$ & $\begin{array}{r}175 \\
1,30 \\
+0,48 \\
\end{array}$ & $\begin{array}{c}135 \\
1 \\
0 \\
\end{array}$ & $\begin{array}{c}89 \\
0,66 \\
-0,54 \\
\end{array}$ & $\begin{array}{c}66 \\
0,49 \\
-0,81 \\
\end{array}$ \\
\hline $\begin{array}{l}\text { Chloride Excretion, } \\
\mu \mathrm{M} / 24 \mathrm{~h} \cdot 100 \mathrm{~g} \text { Body Mass }\end{array}$ & $\begin{array}{c}244 \\
1,69 \\
+1,01\end{array}$ & $\begin{array}{c}203 \\
1,41 \\
+0,60\end{array}$ & $\begin{array}{r}195 \\
1,35 \\
+0,52\end{array}$ & $\begin{array}{c}144 \\
1 \\
0 \\
\end{array}$ & $\begin{array}{c}102 \\
0,71 \\
-0,43 \\
\end{array}$ & $\begin{array}{c}66 \\
0,46 \\
-0,80\end{array}$ \\
\hline $\begin{array}{l}\text { Sodium } \\
\text { Urine, } \\
\mathrm{mM} / \mathrm{L}\end{array}$ & $\begin{array}{r}135 \\
1,28 \\
+0,45 \\
\end{array}$ & $\begin{array}{c}128 \\
1,22 \\
+0,34\end{array}$ & $\begin{array}{c}117 \\
1,11 \\
+0,18\end{array}$ & $\begin{array}{c}105 \\
1 \\
0 \\
\end{array}$ & $\begin{array}{c}64 \\
0,61 \\
-0,62 \\
\end{array}$ & $\begin{array}{c}53 \\
0,50 \\
-0,78 \\
\end{array}$ \\
\hline $\begin{array}{l}\text { Chloride } \\
\text { Urine, } \\
\mathrm{mM} / \mathrm{L}\end{array}$ & $\begin{array}{r}129 \\
1,12 \\
+0,17 \\
\end{array}$ & $\begin{array}{c}132 \\
1,15 \\
+0,21 \\
\end{array}$ & $\begin{array}{r}133 \\
1,15 \\
+0,22 \\
\end{array}$ & $\begin{array}{c}115 \\
1 \\
0 \\
\end{array}$ & $\begin{array}{c}69 \\
0,61 \\
-0,56 \\
\end{array}$ & $\begin{array}{c}47 \\
0,41 \\
-0,85 \\
\end{array}$ \\
\hline $\begin{array}{l}\text { Calcium } \\
\text { Plasma, } \\
\mathrm{mM} / \mathrm{L}\end{array}$ & $\begin{array}{r}3,36 \\
1,00 \\
+0,01 \\
\end{array}$ & $\begin{array}{r}2,32 \\
0,69 \\
-1,01 \\
\end{array}$ & $\begin{array}{l}2,57 \\
0,77 \\
-0,76 \\
\end{array}$ & $\begin{array}{c}3,35 \\
1 \\
0 \\
\end{array}$ & $\begin{array}{r}1,88 \\
0,56 \\
-1,44 \\
\end{array}$ & $\begin{array}{c}2,44 \\
0,73 \\
-0,89 \\
\end{array}$ \\
\hline $\begin{array}{l}\text { Diene conjugates } \\
\text { Urine, } \\
\text { E232/mL }\end{array}$ & $\begin{array}{r}2,14 \\
1,15 \\
+0,43\end{array}$ & $\begin{array}{r}1,66 \\
0,89 \\
-0,30\end{array}$ & $\begin{array}{r}1,90 \\
1,03 \\
+0,07\end{array}$ & $\begin{array}{c}1,86 \\
1 \\
0\end{array}$ & $\begin{array}{c}1,70 \\
0,92 \\
-0,23 \\
\end{array}$ & $\begin{array}{r}1,45 \\
0,78 \\
-0,61 \\
\end{array}$ \\
\hline $\begin{array}{l}\text { Uric Acid } \\
\text { Plasma, } \\
\mu \mathrm{M} / \mathrm{L}\end{array}$ & $\begin{array}{c}781 \\
1,18 \\
+0,35 \\
\end{array}$ & $\begin{array}{c}935 \\
1,41 \\
+0,80 \\
\end{array}$ & $\begin{array}{c}550 \\
0,83 \\
-0,33 \\
\end{array}$ & $\begin{array}{c}662 \\
1 \\
0 \\
\end{array}$ & $\begin{array}{c}716 \\
1,08 \\
+0,16 \\
\end{array}$ & $\begin{array}{c}504 \\
0,76 \\
-0,46 \\
\end{array}$ \\
\hline $\begin{array}{l}\text { Potassium Erythrocytes, } \\
\mathrm{mM} / \mathrm{L}\end{array}$ & $\begin{array}{c}90,1 \\
1,04 \\
+0,46\end{array}$ & $\begin{array}{c}85,8 \\
0,99 \\
-0,18\end{array}$ & $\begin{array}{c}88,5 \\
1,02 \\
+0,21\end{array}$ & $\begin{array}{c}87,0 \\
1 \\
0\end{array}$ & $\begin{array}{r}86,9 \\
1,00 \\
-0,02\end{array}$ & $\begin{array}{l}83,9 \\
0,96 \\
-0,45\end{array}$ \\
\hline $\begin{array}{l}\text { Sodium } \\
\text { Erythrocytes, } \\
\mathrm{mM} / \mathrm{L}\end{array}$ & $\begin{array}{r}25,5 \\
1,16 \\
+0,78\end{array}$ & $\begin{array}{r}22,7 \\
1,03 \\
+0,16\end{array}$ & $\begin{array}{r}21,8 \\
0,99 \\
-0,04 \\
\end{array}$ & $\begin{array}{c}22,0 \\
1 \\
0\end{array}$ & $\begin{array}{r}23,7 \\
1,08 \\
+0,38\end{array}$ & $\begin{array}{c}20,6 \\
0,93 \\
-0,33 \\
\end{array}$ \\
\hline $\begin{array}{l}\text { Canalicular } \\
\text { Reabsorbtion, } \\
\%\end{array}$ & $\begin{array}{r}98,8 \\
1,00 \\
+0,11 \\
\end{array}$ & $\begin{array}{c}99,0 \\
1,00 \\
+0,33 \\
\end{array}$ & $\begin{array}{r}98,9 \\
1,00 \\
+0,24 \\
\end{array}$ & $\begin{array}{c}98,7 \\
1 \\
0 \\
\end{array}$ & $\begin{array}{r}98,7 \\
1,00 \\
-0,02 \\
\end{array}$ & $\begin{array}{c}98,5 \\
1,00 \\
-0,27 \\
\end{array}$ \\
\hline $\begin{array}{l}\text { Potassium Excretion, } \\
\mu \mathrm{M} / 24 \mathrm{~h} \cdot 100 \mathrm{~g} \text { Body Mass }\end{array}$ & $\begin{array}{c}197 \\
1,04 \\
+0,07 \\
\end{array}$ & $\begin{array}{r}173 \\
0,92 \\
-0,13 \\
\end{array}$ & $\begin{array}{r}189 \\
1,00 \\
+0,01 \\
\end{array}$ & $\begin{array}{c}189 \\
1 \\
0 \\
\end{array}$ & $\begin{array}{c}191 \\
1,01 \\
+0,02 \\
\end{array}$ & $\begin{array}{c}179 \\
0,95 \\
-0,08 \\
\end{array}$ \\
\hline Pattern III (12) & $\begin{array}{l}+0,48 \\
\pm 0,14\end{array}$ & $\begin{array}{l}+0,20 \\
\pm 0,16\end{array}$ & $\begin{array}{l}+0,09 \\
\pm 0,10\end{array}$ & 0 & $\begin{array}{l}-0,33 \\
\pm 0,14\end{array}$ & $\begin{array}{l}-0,61 \\
\pm 0,09\end{array}$ \\
\hline
\end{tabular}


https://doi.org/

\begin{tabular}{|c|c|c|c|c|c|c|}
\hline $\begin{array}{l}\text { Calcium } \\
\text { Urine, } \\
\mathrm{mM} / \mathrm{L}\end{array}$ & $\begin{array}{c}1,93 \\
0,92 \\
-0,44 \\
\end{array}$ & $\begin{array}{c}2,32 \\
1,11 \\
+0,59 \\
\end{array}$ & $\begin{array}{c}2,07 \\
0,99 \\
-0,07 \\
\end{array}$ & $\begin{array}{c}2,10 \\
1 \\
0\end{array}$ & $\begin{array}{r}2,15 \\
1,02 \\
+0,13 \\
\end{array}$ & $\begin{array}{c}3,05 \\
1,46 \\
+2,55 \\
\end{array}$ \\
\hline $\begin{array}{l}\text { Calcium Excretion, } \\
\mu \mathrm{M} / 24 \mathrm{~h} \cdot 100 \mathrm{~g} \text { Body Mass }\end{array}$ & $\begin{array}{c}4,50 \\
1,55 \\
+1,05\end{array}$ & $\begin{array}{c}4,25 \\
1,47 \\
+0,88\end{array}$ & $\begin{array}{c}3,09 \\
1,07 \\
+0,13\end{array}$ & $\begin{array}{c}2,90 \\
1 \\
0\end{array}$ & $\begin{array}{r}3,08 \\
1,06 \\
+0,12\end{array}$ & $\begin{array}{c}4,76 \\
1,64 \\
+1,22\end{array}$ \\
\hline $\begin{array}{l}\text { Urea } \\
\text { Plasma, } \\
\mathrm{mM} / \mathrm{L}\end{array}$ & $\begin{array}{c}7,44 \\
1,00 \\
+0,01\end{array}$ & $\begin{array}{c}7,85 \\
1,06 \\
+0,25\end{array}$ & $\begin{array}{c}9,29 \\
1,25 \\
+1,09\end{array}$ & $\begin{array}{c}7,42 \\
1 \\
0\end{array}$ & $\begin{array}{c}8,92 \\
1,20 \\
+0,88\end{array}$ & $\begin{array}{c}10,03 \\
1,35 \\
+1,52\end{array}$ \\
\hline $\begin{array}{l}\text { Katalase } \\
\text { Plasma, } \\
\mathrm{nM} / \mathrm{h} \cdot \mathrm{mL}\end{array}$ & $\begin{array}{c}135 \\
1,30 \\
+1,12\end{array}$ & $\begin{array}{c}122 \\
1,18 \\
+0,66 \\
\end{array}$ & $\begin{array}{c}120 \\
1,16 \\
+0,60\end{array}$ & $\begin{array}{c}103 \\
1 \\
0 \\
\end{array}$ & $\begin{array}{c}145 \\
1,40 \\
+1,49\end{array}$ & $\begin{array}{c}142 \\
1,37 \\
+1,38 \\
\end{array}$ \\
\hline $\begin{array}{l}\text { Creatinine } \\
\text { Plasma, } \\
\mu \mathrm{M} / \mathrm{L}\end{array}$ & $\begin{array}{c}73 \\
1,01 \\
-0,04 \\
\end{array}$ & $\begin{array}{c}68 \\
0,94 \\
-0,18 \\
\end{array}$ & $\begin{array}{c}90 \\
1,24 \\
+0,72 \\
\end{array}$ & $\begin{array}{c}73 \\
1 \\
0 \\
\end{array}$ & $\begin{array}{c}86 \\
1,18 \\
+0,55 \\
\end{array}$ & $\begin{array}{c}98 \\
1,35 \\
+1,04 \\
\end{array}$ \\
\hline $\begin{array}{l}\text { Cholesterol } \\
\text { Plasma, } \\
\mathrm{mM} / \mathrm{L}\end{array}$ & $\begin{array}{c}1,46 \\
0,93 \\
-0,23 \\
\end{array}$ & $\begin{array}{c}1,53 \\
0,97 \\
-0,09 \\
\end{array}$ & $\begin{array}{r}1,62 \\
1,03 \\
+0,11 \\
\end{array}$ & $\begin{array}{c}1,57 \\
1 \\
0 \\
\end{array}$ & $\begin{array}{c}1,60 \\
1,02 \\
+0,06 \\
\end{array}$ & $\begin{array}{r}1,67 \\
1,06 \\
+0,21 \\
\end{array}$ \\
\hline $\begin{array}{l}\text { Amylase } \\
\text { Urine, } \\
\mathrm{mg} / \mathrm{h} \cdot \mathrm{mL}\end{array}$ & $\begin{array}{c}181 \\
0,90 \\
-0,39 \\
\end{array}$ & $\begin{array}{c}215 \\
1,06 \\
+0,23 \\
\end{array}$ & $\begin{array}{c}210 \\
1,04 \\
+0,14\end{array}$ & $\begin{array}{c}202 \\
1 \\
0\end{array}$ & $\begin{array}{c}212 \\
1,05 \\
+0,18\end{array}$ & $\begin{array}{r}210 \\
1,04 \\
+0,15 \\
\end{array}$ \\
\hline Pattern IV (7) & $\begin{array}{l}+0,15 \\
\pm 0,25\end{array}$ & $\begin{array}{l}+0,33 \\
\pm 0,15\end{array}$ & $\begin{array}{l}+0,39 \\
\pm 0,16\end{array}$ & 0 & $\begin{array}{l}+0,49 \\
\pm 0,20\end{array}$ & $\begin{array}{l}+1,15 \\
\pm 0,31\end{array}$ \\
\hline
\end{tabular}

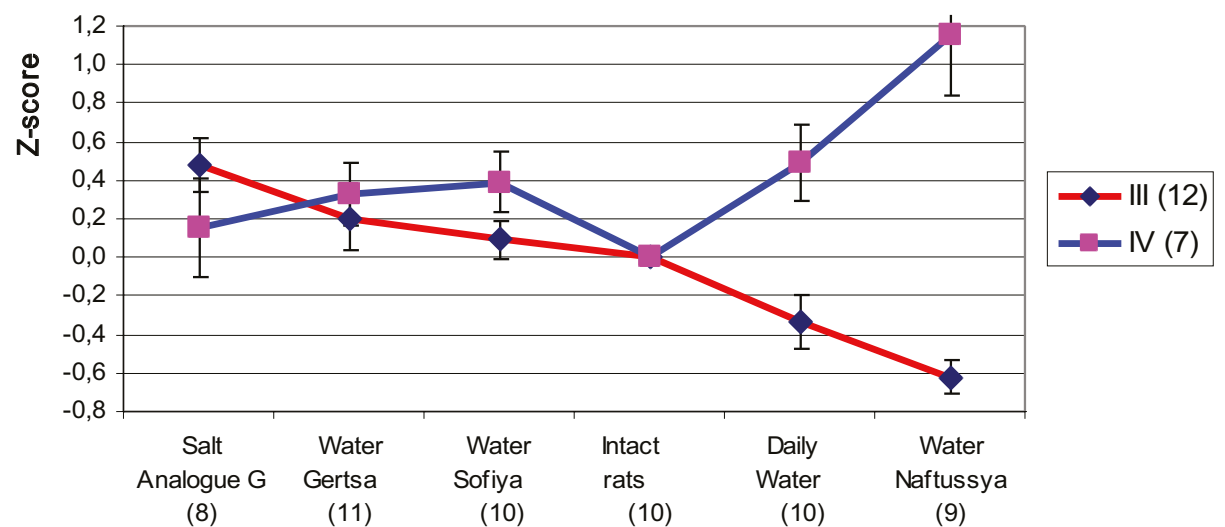

Fig. 2. The second pair of metabolic parameters of reactions to water-salt loads.

The fifth pattern (Table 4 and Fig. 3) combines 6 parameters, the mean value $\mathrm{Z}$ of which increases significantly under the influence of "Sofiya" water. Urinary excretion, concentration of magnesium and creatinine in urine and plasma amylase activity are the most prevalent, while phosphatemia and urine concentration of malonic dialdehyde are slightly increased. Under the influence of "Naftussia" water the average $\mathrm{Z}$ value tends to increase, whereas neither 'Gertsa' water nor its salt analogue causes significant changes in these parameters, while daily water causes a weak tendency to decrease them.

The sixth pattern, mirrored to the fifth, reflects the maximum drop in plasma chloride and middle mass molecules levels, urinary concentration, excretion of uric acid and the tendency towards decreasing the level of malonic dialdehyde, sodium and magnesium in the plasma. Instead, these parameters increase to varying degrees under the influence of tap water, not reacting substantially to "Gertsa" water or to its salt analogue. 
Table 4

The third pair of metabolic parameters of reactions to water-salt loadings

\begin{tabular}{|c|c|c|c|c|c|c|}
\hline Variables & $\begin{array}{c}\text { Daily } \\
\text { Water } \\
(10)\end{array}$ & $\begin{array}{c}\text { MW } \\
\text { Gertsa } \\
(11)\end{array}$ & $\begin{array}{c}\text { Intact } \\
\text { rats } \\
(10)\end{array}$ & $\begin{array}{l}\text { Salt } \\
\text { Anal } \\
\text { G (8) }\end{array}$ & $\begin{array}{c}\text { Naftu } \\
\text { ssya } \\
\text { (9) }\end{array}$ & $\begin{array}{c}\text { MW } \\
\text { Sofiya } \\
\text { (10) }\end{array}$ \\
\hline $\begin{array}{l}\text { Magnesium Excretion, } \\
\mu \mathrm{M} / 24 \mathrm{~h} \cdot 100 \text { g Body Mass }\end{array}$ & $\begin{array}{r}2,65 \\
0,80 \\
-0,31\end{array}$ & $\begin{array}{c}2,51 \\
0,76 \\
-0,38\end{array}$ & $\begin{array}{c}3,30 \\
1 \\
0\end{array}$ & $\begin{array}{c}5,85 \\
1,77 \\
+1,23\end{array}$ & $\begin{array}{c}5,07 \\
1,54 \\
+0,85\end{array}$ & $\begin{array}{c}5,98 \\
1,81 \\
+1,29\end{array}$ \\
\hline $\begin{array}{l}\text { Magnesium } \\
\text { Urine, } \\
\text { mM/L }\end{array}$ & $\begin{array}{c}1,90 \\
0,74 \\
-0,37 \\
\end{array}$ & $\begin{array}{l}1,73 \\
0,68 \\
-0,47 \\
\end{array}$ & $\begin{array}{c}2,56 \\
1 \\
0 \\
\end{array}$ & $\begin{array}{c}2,54 \\
0,99 \\
-0,01 \\
\end{array}$ & $\begin{array}{c}3,27 \\
1,26 \\
+0,38 \\
\end{array}$ & $\begin{array}{c}4,09 \\
1,60 \\
+0,86 \\
\end{array}$ \\
\hline $\begin{array}{l}\text { Creatinine } \\
\text { Urine, } \\
\mathrm{mM} / \mathrm{L}\end{array}$ & $\begin{array}{c}7,15 \\
1,12 \\
+0,40\end{array}$ & $\begin{array}{c}6,83 \\
1,07 \\
+0,23 \\
\end{array}$ & $\begin{array}{c}6,41 \\
1 \\
0\end{array}$ & $\begin{array}{r}7,01 \\
1,09 \\
+0,32 \\
\end{array}$ & $\begin{array}{c}7,16 \\
1,12 \\
+0,41 \\
\end{array}$ & $\begin{array}{c}8,12 \\
1,27 \\
+0,93 \\
\end{array}$ \\
\hline $\begin{array}{l}\text { Amylase } \\
\text { Plasma, } \\
\mathrm{mg} / \mathrm{h} \cdot \mathrm{mL}\end{array}$ & $\begin{array}{c}145 \\
0,96 \\
-0,27\end{array}$ & $\begin{array}{c}163 \\
1,07 \\
+0,45\end{array}$ & $\begin{array}{c}152 \\
1 \\
0\end{array}$ & $\begin{array}{c}134 \\
0,88 \\
-0,73\end{array}$ & $\begin{array}{c}152 \\
1,00 \\
-0,01\end{array}$ & $\begin{array}{c}171 \\
1,13 \\
+0,78\end{array}$ \\
\hline $\begin{array}{l}\text { Phosphate } \\
\text { Plasma, } \\
\mathrm{mM} / \mathrm{L}\end{array}$ & $\begin{array}{c}0,87 \\
0,84 \\
-0,27 \\
\end{array}$ & $\begin{array}{c}0,72 \\
0,69 \\
-0,52 \\
\end{array}$ & $\begin{array}{c}1,04 \\
1 \\
0\end{array}$ & $\begin{array}{c}0,92 \\
0,88 \\
-0,20 \\
\end{array}$ & $\begin{array}{c}0,88 \\
0,85 \\
-0,26 \\
\end{array}$ & $\begin{array}{c}1,22 \\
1,18 \\
+0,30 \\
\end{array}$ \\
\hline $\begin{array}{l}\text { Malonic Dialdehyde Urine, } \\
\mu \mathrm{M} / \mathrm{L}\end{array}$ & $\begin{array}{c}77 \\
0,83 \\
-0,36 \\
\end{array}$ & $\begin{array}{c}91 \\
0,99 \\
-0,03 \\
\end{array}$ & $\begin{array}{c}92 \\
1 \\
0 \\
\end{array}$ & $\begin{array}{c}81 \\
0,88 \\
-0,25 \\
\end{array}$ & $\begin{array}{c}87 \\
0,95 \\
-0,11 \\
\end{array}$ & $\begin{array}{c}102 \\
1,10 \\
+0,22 \\
\end{array}$ \\
\hline Pattern V (6) & $\begin{array}{l}-0,20 \\
\pm 0,12\end{array}$ & $\begin{array}{l}-0,12 \\
\pm 0,16\end{array}$ & 0 & $\begin{array}{l}+0,06 \\
\pm 0,27\end{array}$ & $\begin{array}{l}+0,21 \\
\pm 0,17\end{array}$ & $\begin{array}{l}+0,78 \\
\pm 0,17\end{array}$ \\
\hline $\begin{array}{l}\text { Chloride } \\
\text { Plasma, } \\
\mathrm{mM} / \mathrm{L}\end{array}$ & $\begin{array}{c}95,0 \\
1,01 \\
+0,20\end{array}$ & $\begin{array}{c}91,5 \\
0,98 \\
-0,38\end{array}$ & $\begin{array}{c}93,8 \\
1 \\
0\end{array}$ & $\begin{array}{c}92,9 \\
0,99 \\
-0,14\end{array}$ & $\begin{array}{c}93,2 \\
0,99 \\
-0,10\end{array}$ & $\begin{array}{c}89,7 \\
0,96 \\
-0,67\end{array}$ \\
\hline $\begin{array}{l}\text { Middle Mass Molecules } \\
\text { Plasma, } \\
\text { units }\end{array}$ & $\begin{array}{r}193 \\
1,25 \\
+0,76 \\
\end{array}$ & $\begin{array}{c}119 \\
0,78 \\
-0,67 \\
\end{array}$ & $\begin{array}{c}154 \\
1 \\
0\end{array}$ & $\begin{array}{c}148 \\
0,96 \\
-0,11 \\
\end{array}$ & $\begin{array}{c}134 \\
0,87 \\
-0,38 \\
\end{array}$ & $\begin{array}{c}126 \\
0,82 \\
-0,55 \\
\end{array}$ \\
\hline $\begin{array}{l}\text { Uric Acid } \\
\text { Urine, } \\
\mathrm{mM} / \mathrm{L}\end{array}$ & $\begin{array}{r}4,70 \\
1,28 \\
+0,55 \\
\end{array}$ & $\begin{array}{c}4,23 \\
1,15 \\
+0,30 \\
\end{array}$ & $\begin{array}{c}3,68 \\
1 \\
0 \\
\end{array}$ & $\begin{array}{l}2,91 \\
0,79 \\
-0,42 \\
\end{array}$ & $\begin{array}{l}3,18 \\
0,86 \\
-0,27 \\
\end{array}$ & $\begin{array}{c}2,56 \\
0,69 \\
-0,61 \\
\end{array}$ \\
\hline $\begin{array}{l}\text { Uric Acid Excretion, } \\
\mu \mathrm{M} / 24 \mathrm{~h} \cdot 100 \mathrm{~g} \text { Body Mass }\end{array}$ & $\begin{array}{c}6,7 \\
1,16 \\
+0,18\end{array}$ & $\begin{array}{c}6,0 \\
1,04 \\
+0,04\end{array}$ & $\begin{array}{c}5,7 \\
1 \\
0\end{array}$ & $\begin{array}{c}6,5 \\
1,14 \\
+0,15\end{array}$ & $\begin{array}{c}4,9 \\
0,86 \\
-0,15\end{array}$ & $\begin{array}{c}3,8 \\
0,66 \\
-0,36\end{array}$ \\
\hline $\begin{array}{l}\text { Malonic Dialdehyde } \\
\text { Plasma, } \\
\mu \mathrm{M} / \mathrm{L}\end{array}$ & $\begin{array}{c}92 \\
1,45 \\
+1,30 \\
\end{array}$ & $\begin{array}{c}81 \\
1,28 \\
+0,83 \\
\end{array}$ & $\begin{array}{c}63 \\
1 \\
0 \\
\end{array}$ & $\begin{array}{c}62 \\
0,97 \\
-0,08 \\
\end{array}$ & $\begin{array}{c}80 \\
1,26 \\
+0,76 \\
\end{array}$ & $\begin{array}{c}57 \\
0,91 \\
-0,27 \\
\end{array}$ \\
\hline $\begin{array}{l}\text { Sodium } \\
\text { Plasma, } \\
\mathrm{mM} / \mathrm{L}\end{array}$ & $\begin{array}{c}131,8 \\
1,03 \\
+0,40 \\
\end{array}$ & $\begin{array}{c}128,6 \\
1,00 \\
+0,01 \\
\end{array}$ & $\begin{array}{c}128,6 \\
1 \\
0 \\
\end{array}$ & $\begin{array}{l}127,8 \\
0,99 \\
-0,09 \\
\end{array}$ & $\begin{array}{c}129,9 \\
1,01 \\
+0,16 \\
\end{array}$ & $\begin{array}{c}127,0 \\
0,99 \\
-0,19 \\
\end{array}$ \\
\hline $\begin{array}{l}\text { Magnesium } \\
\text { Plasma, } \\
\mathrm{mM} / \mathrm{L}\end{array}$ & $\begin{array}{r}1,05 \\
1,19 \\
+0,28 \\
\end{array}$ & $\begin{array}{c}0,70 \\
0,80 \\
-0,29 \\
\end{array}$ & $\begin{array}{c}0,88 \\
1 \\
0 \\
\end{array}$ & $\begin{array}{c}0,79 \\
0,89 \\
-0,16 \\
\end{array}$ & $\begin{array}{c}0,90 \\
1,02 \\
+0,03\end{array}$ & $\begin{array}{c}0,80 \\
0,90 \\
-0,14\end{array}$ \\
\hline Pattern VI (7) & $\begin{array}{l}+0,52 \\
\pm 0,15\end{array}$ & $\begin{array}{l}-0,02 \\
\pm 0,19\end{array}$ & 0 & $\begin{array}{l}-0,12 \\
\pm 0,07\end{array}$ & $\begin{array}{l}+0,01 \\
\pm 0,14\end{array}$ & $\begin{array}{l}-0,40 \\
\pm 0,08\end{array}$ \\
\hline
\end{tabular}

At the next stage of the analysis, three pairs of mirror patterns have been doubled and modulated by the reaction of the parameters to the dominant stimulus (Fig. 4). 


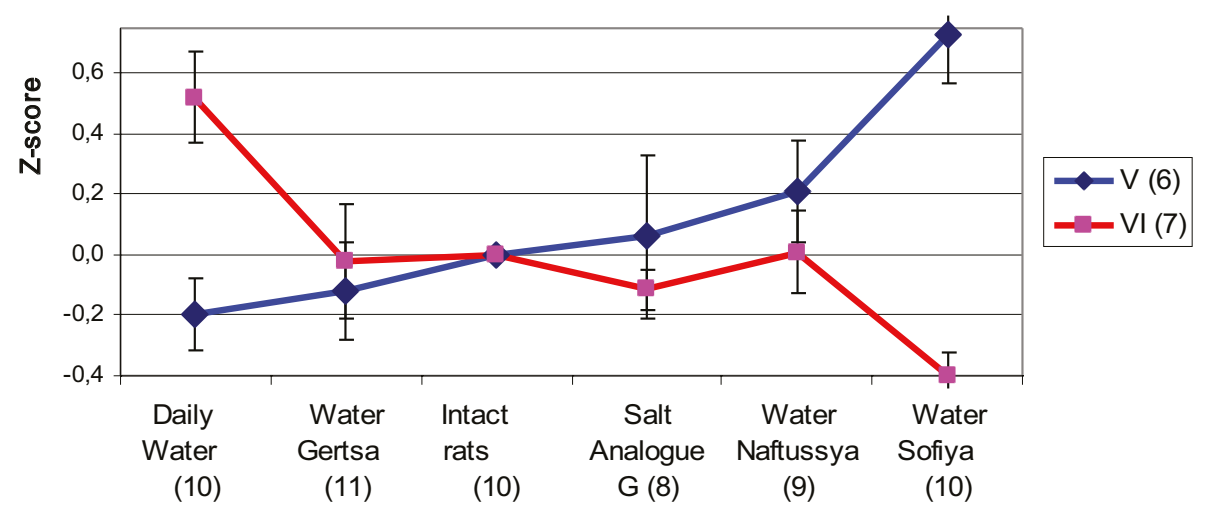

Fig. 3. The third pair of metabolic parameters of reactions to water-salt loads.

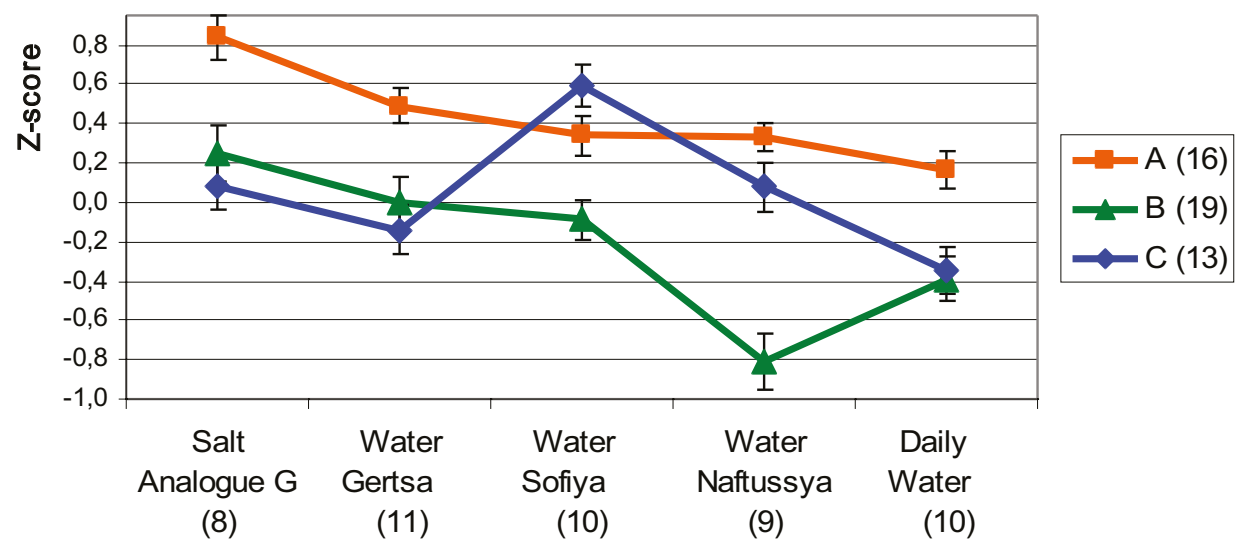

Fig. 4. Doubles and modulated super patterns of metabolic parameters of reactions to water-salt loads.

16 parameters have composed a superpattern A; that deviates from the maximum level in the intact rats because of the influence of the salt analogue of "Gertsa" water; it has weaker, but also tangible effect made by "Gertsa" native water and even slighter effect made by the water "Sofiya" and "Naftussia", while tap water is almost ineffective in relation to these metabolic parameters. It seems that the deviation of these parameters of metabolism from the control is most influenced by the cations of $\mathrm{Na}^{+}$and $\mathrm{Cl}^{-}$entering the body, while the simultaneous receipt of trace elements and organic carbon, and possibly of sulphate anion, weakens the effect of $\mathrm{NaCl}$, whereas organic nitrogen enhances it.

The other 19 parameters have composed a superpattern $\mathrm{B}$, that deviate to a maximum extent from the reference level after the use of water "Naftussya"; tap water is less effective, whereas quasi-isotonic liquids are almost inactive if to talk about these parameters. Apparently, their deviation is caused by stress during the process of water loading, as well as hypotonicity of water. Organic substances deepen the deviation, whereas the quasi-isotonicity of water reduces them.

The other 13 parameters of a superpattern $\mathrm{C}$ in animals that have been given tap water, deviate from intact control to the same extent as in the previous superpattern, which, apparently, is also due to the stressful effects of the load course. Both "Naftussia" and "Gertsa" water and its salt analogue prevent the stress deviations of these parameters. Instead, consumption of water "Sofiya" doesn't prevent the stress deviations of these parameters, which is apparently 
due to the presence of its composition sulfate and organic nitrogen, the presence of which in the other liquids is negligible.

Conclusion. The features of the reactions of the parameters of metabolism are caused by $\mathrm{NaCl}, \mathrm{SO}_{4}^{2-}$, organic carbon and nitrogen in water.

\title{
Conformity to ethical standards.
}

Experiments on animals have been carried out in accordance with the provisions of the Helsinki Declaration of 1975, revised and supplemented in 2002 by the Directives of the National Committees for Ethics in Scientific Research.

The conduct of experiments was approved by the Ethics Committee of the Horbachevskyi Ternopil' State Medical University. The modern rules for the maintenance and use of laboratory animals complying with the principles of the European Convention for the Protection of Vertebrate Animals used for scientific experiments and needs are observed (Strasbourg, 1985).

Рекомендовано до друку комісією з біоетики

\section{REFERENCES}

1. Bilas VR, Popovych IL. Role of microflora and organic substances of water Naftussya in its modulating influence on neuroendocrine-immune complex and metabolism. Medical Hydrology and Rehabilitation. 2009;7(1):68-102. 2. Chebanenko OI, Chebanenko LO, Popovych $I L$. Variety Balneoeffects of Factors Spa Truskavets' and their Forecast. K.: UNESCO-SOCIO; 2012. 496. 3. Chebanenko OI, Flyunt IS, Popovych IL, Balanovs'kyi VP, Lakhin PV. Water Naftussya and hydro-mineral exchange. K.: Nauk. dumka; 1997. 141. 4. Goryachkovskiy AM. Clinical Biochemistry [in Russian]. Odesa: Astroprint; 1998. 608. 5. Gozhenko AI, Dolomatov SI, Shumiliva PA, Topor EA, Pyatenko VA, Badiin IYu. The effect of osmotic stresses on the functional state of healthy kidneys [in Russian]. Nefrologiya. 2004;8(2):44-8. 6. Ivassivka SV. Biological Active Substances of Water Naftussya, their Genesis and Mechanisms of Physiological Effects. K.: Nauk. dumka; 1997. 110. 7. Ivassivka SV, Bubnyak AB, Kovbasnyuk MM, Popovych IL. Genesis and role of phenols in waters from Naftussya layer. In: Problems of pathology in experiment and clinic. Scientific works of Drohobych Medical Institute. Vol. XV. Drohobych; 1994.6-11. 8. Ivassivka SV, Popouych IL, Aksentijchuk BI, Bilas VR. Nature of Balneofactors of Water Naftussya and Essence its Curative and Prophilactive Effects. Truskavets': Truskavets'kurort; 1999. 125. 9. Ivassivka SV, Popovych IL, Aksentijchuk BI, Flyunt IS. Physiological Activity of Uric Acid and its Role in the Mechanism of Action of Water Naftussya. K.: Computerpress; 2004. 163. 10. Kozyavkina OV, Kozyavkina NV, Gozhenko OA, Gozhenko AI, Barylyak LG., Popovych $I L$. Bioactive Water Naftussya and Neuro-Endocrine-Immune Complex. K.: UNESCO-SOCIO; 2015. 349. 11. Popovych IL, Ivassivka SV. Role of organic substances of water Naftussya in its physiological activity. Medical Hydrology and Rehabilitation. 2009;7(2):6-26. 12. Popovych IL, Vis'tak HI, Gumega MD, Ruzhylo SV. Vegetotropic Effects of Bioactive Water Naftussya and their Endocrine-Immune, Metabolic and Hemodynamic Accompaniments. K.: UNESCO-SOCIO; 2014. 163. 13. Yaremenko MS, Ivassivka SV, Popovych AI, Bilas VR et al. Physiological Bases of Curative Effect of Water Naftussya. K.: Nauk. dumka; 1989. 144. 14. Zavidnyuk YV, Mysula IR, Klishch IM, Zukow W, Popovych IL, Korda MM. General non-specific metabolic, neuroendocrine and immune reactions to various water-salt loads in female rats. Journal of Education, Health and Sport. 2018;8(3):513-24.

Стаття надійшла до редколегії 28.05.2018

\section{RESEARCH ARTICLE}

\section{Features of Metabolic Reactions to Various Water-Salt Loads in the Female Rats}

\author{
Y.V. ZAVIDNYUK \\ IY Horbachevs'kyi State Medical University, Ternopil', Ukraine \\ Ukrainian Scientific Research Institute of Transport Medicine, Odesa, Ukraine \\ E-mail: zavidnyukyv@tdmu.edu.ua
}

Background. In the previous article it has been described that the registered parameters of water-salt, nitrous and lipid metabolism as well as the neuroendocrine-immune complex found in 42 rats subjected to various water-salt loads, significantly differ from the parameters of the intact rats; the groups of animals have received the liquids of different mineralization and 
chemical composition. The article aims at finding out the features of the reaction parameters of metabolism.

Materials and methods. Experiment has been performed at 58 healthy female Wistar rats 240-290 g divided into 6 groups. Animals of the first group have been intact, using tap water from drinking ad libitum. Instead, the other rats have received the same tap water as well as water of such trade marks as "Sophiya", "Naftussia", "Gertsa" and its artificial salt analogue through the probe at a dose of 1,5 mL/100 g of body mass for 6 days. The day after the completion of the drinking course the parameters of water-salt, nitrous and lipid metabolism have been registered.

Results. It has been found that 16 metabolic parameters deviate from those in the intact rats, what has been caused by the salt analogue of "Gertsa" water; "Gertsa" native water has made a slighter, but also tangible effect, and "Sofiya" and "Naftussia" trademarks of water have made the smallest influence, instead of ordinary water is almost ineffective in relation to these metabolic parameters. Other 19 parameters deviate from the reference level after the use of water Naftussya, fresh water is less effective, whereas quasi-isotonic liquids are almost inactive of to talk about these parameters. Other 13 parameters of the animals that use normal water as deviate from the intact control as in the previous super pattern, that is also caused by the stressful effects of the load course. Both "Naftussia" and "Gertsa" water and its salt analogue prevent the stress deviations of these parameters. Instead, consumption of water "Sofiya" shows that deviations of these parameters are reversed.

Conclusion. The features of the reactions of the parameters of metabolism are caused by a level of $\mathrm{NaCl}, \mathrm{SO}_{4}{ }^{2-}$ as well as organic carbon and nitrogen in water.

Key words: water-salt loads, water-salt, nitrous and lipid metabolism, female rats. 